\title{
Valoración de la actividad objetal en una muestra de niños mexicanos de 13 a 52 meses
}

\section{Assessment of actions with objects in Mexican children from 13 to 52 months old}

\author{
Solovieva, Yulia ${ }^{1}$; García Flores, Marco Antonio ${ }^{1}$; Quintanar Rojas, Luis ${ }^{1}$ y Moreno Agundis \\ Saraí ${ }^{1}$
}

\begin{abstract}
Resumen:
La adquisición de acciones objetales constituye la actividad rectora en la edad preescolar temprana. Actualmente, en la práctica psicológica no se cuenta con instrumentos de evaluación de la adquisición de las acciones objetales en forma de interacción cualitativa significativa para esta edad. El objetivo de artículo consiste en presentar datos de evaluación de estas acciones en niños entre 13 y 52 meses de edad a partir de un instrumento cualitativo "Evaluación de acciones objetales en preescolares menores" de Solovieva y Quintanar (2014). Los resultados permiten establecer una dinámica cualitativa de las etapas de adquisición de las acciones con objetos. En particular, se precisaron las etapas de adquisición de acciones con objetos y juguetes con el paso al inicio de la adquisición de la función simbólica con el rol específico de uso de juguetes animados. Se discute la utilidad del instrumento cualitativo para el diagnóstico psicológico temprano y organización de la interacción con niños preescolares, así como para la formulación de programas de intervención y desarrollo en esta edad.
\end{abstract}

Palabras Clave: edad preescolar, desarrollo, actividad objetal, acción simbólica, juego temprano.

\begin{abstract}
:
Acquisition of actions with objects corresponds to dominant activity of early preschool childhood. Psychological practice doesn't count with specific instruments for assessment of acquisition of actions with objects within qualitative interaction meaningful for this age. The objective of the article is to present the data of assessment of such actions in children between 13 and 52 months old. For the interactions with children, new qualitative instrument of assessment "Evaluation of objection actions in preschool children" was used (Solovieva and Quintanar, 2014). The results of the study allowed to establish specific qualitative stages of development of actions with concrete objects and toys. Particularly, the stages of initial appearance of symbolic actions with an important role of animated toys were found. The authors discuss the usefulness of qualitative instruments and procedures for assessment of early psychological development and for interaction with children together with the possibility of creation of programs for correction of children at this age.
\end{abstract}

Keywords: preschool age, development, object activity, symbolic action, early play.

\footnotetext{
${ }^{1}$ Benemerita Universidad Autónoma de Puebla. Facultad de Psicología, Puebla, México.

*Correspondencia: yulia.solovieva@correo.buap.mx.
} 
La adquisición de las acciones objetales o actividad instrumental constituye la línea central del desarrollo entre el primer y tercer año de edad. Estas acciones se refieren al dominio de los procedimientos, socialmente elaborados, de acción con los objetos con la participación de los adultos (Elkonin, 1987; McCune-Nicolich, 1995). El tema de adquisición de las acciones objetales es poco común en psicología del desarrollo, debido a que la mayor atención se le da al desarrollo verbal o comunicativo e intelectual (Piaget, 1992; Piaget y Inhelder, 1997; Slade, 1987). Se considera que, debido a la relación y comunicación con los adultos, los niños van comprendiendo la significación social de los objetos (Cruz, 1995; Bates, Benigni, Bretherton, Camaioni \& Volterra, 1979; Tomasello, 2013; Moro y Rodríguez, 1991) y poco a poco van adquiriendo lo técnico operacional (uso del objeto). Sin embargo, es imporante considerar, que la comunicación emocional inespecífica entre el niño y adulto se enriquece no sólo gracias al lenguaje, sino también por el uso de objetos culturales disponibles en el entorno del niño.

Elkonin opina que el uso de objetos de la vida cotidiana surge en aquellas situaciones, cuando el niño, al tener un mejor manejo exitoso de las herramientas, obtiene el estímulo y la alabanza del adulto (Elkonin, 1980). Lo anterior permite establecer el puente entre la edad psicológica temprana comunicativo emocional y la edad preescolar temprana, en la cual predomina la posibilidad de adquisición de las acciones objetales, apoyada en un contacto afectivo emocional positivo con el adulto. Podemos suponer que, la previa edad psicológica, en la cual predomina la comunicación afectiva con el adulto (Lisina, 1986), garantiza el fondo emocional positivo para la actividad objetal futura. Gradualmen- te, en el niño se forma lo que muchos autores llaman "la inteligencia práctica", en la cual el niño inicia a dominar las operaciones objetales-instrumentales (Elkonin, 1987), y también tiene lugar la consolidación de los esquemas sensoriomotores que preparan el surgimiento de la función simbólica (Piaget, 1992; Piaget y Inhelder, 1997).

Gracias al uso de los objetos (acciones del niño que tienen un objetivo y sentido cultural preciso), el niño logra generalizar las características perceptivas de los objetos concretos a través de su funcionalidad y, de esta forma, adquiere la imagen interna de dichos objetos (Galperin, 2009a, 2009b). El uso de objetos en forma de acciones funcionales y el juego objetal le permite al niño lograr una generalización de dominio de los objetos en forma práctica en diversas situaciones y desplegar secuencias de acciones relacionadas con un tema. En el momento inicial de este proceso predomina en el infante la relación objeto-acción sobre el significado, pero cuando un objeto sustituye al otro y se realiza la misma acción de forma representativa en lugar de la práctica, la relación se invierte: predomina, entonces, el significado sobre el objeto. De esta forma, la acción concreta se convierte en la acción simbólica (Vigotsky, 1979).

Lo anterior se evidencia en la capacidad del niño de representar acciones ante ausencia de objeto y en la aparición de cadenas de acciones representativas que llamamos juego simbólico desde los 2 años de edad aproximadamente (Bonilla, Solovieva y Jiménez, 2012). Gradualmente, en el juego, el niño sustituye un objeto por otro y realiza las acciones representativas correspondientes combinadas con las prácticas. De esta manera el objeto y la acción pierden su carácter únicamente con- 
creto, lo cual permite la generalización del aspecto técnico operacional y del significado verbal y no verbal.

En las acciones objetales y, posteriormente, en el juego simbólico, se da la identificación espontánea de las características de los objetos, el reconocimiento y la evocación de los mismos, las acciones y las situaciones ante y sin su presencia (del objeto) y la reproducción y secuencias de acciones en diversas situaciones (Solovieva y Quintanar, 2016a).

Al término de esta etapa (Davidov, 1988) las formaciones psicológicas nuevas que surgen en la vida psíquica del niño son los significados verbales concretos, la designación del sentido a las cosas, la percepción categorial generalizada del mundo objetal y el pensamiento concreto en acciones. La edad de acciones objetales, entonces, se caracteriza por su orientación hacia el conocimiento del mundo externo cultural (Lisina, 1986).

En la psicología del desarrollo se ha notado que la ausencia de acciones objetales en la infancia puede tener repercusiones negativas en el desarrollo del lenguaje, en los movimientos voluntarios y en general en todas las funciones psicológicas que surgen gracias a la actividad práctica (Liublinskaia, 1971).

Sobre la base de las acciones objetales se adquiere la función simbólica compleja, mientras que la ausencia y la pobreza de las acciones objetales implica obstáculos para dicha función (Salmina, 2010). A su vez, la pobreza simbólica imposibilita la inclusión del niño en la actividad de juego temático de roles. Es sus inicios, el juego de roles se basa en una simbolización primaria o aceptación del rol (Lisina, 1986). Posteriormente, esta función se desarrolla en el nivel complejo, cual implica la explicación reflexiva y consciente de diversas situaciones, en las que se usan signos y símbolos (González \& Solo- vieva, 2014). Gracias a la función simbólica, el niño forma el conocimiento generalizado y abstracto del mundo y, por ende, se conforma el pensamiento en conceptos (Vigotsky, 1979).

La función simbólica surge como una sustitución de un objeto por otro solo sobre la base de las acciones objetales ya adquiridas, es decir, cuando se le da un significado distinto $\mathrm{y}$ novedoso a algo que es ya conocido $\mathrm{y}$ que se puede realizar en distintos niveles de la acción: materializado, perceptivo y verbal (González y Solovieva, 2015, 2016). Lo anterior, sin duda alguna, se relaciona con una iniciativa que procede del niño y que gradualmente se hace cada vez más reflexiva.

En muchas ocasiones, en estudios psicológicos, no se hace distinción entre la acción objetal que implica el uso apropiado de un objeto cultural (tomar taza vacía o darle a un muñeco a beber) y una acción simbólica que sustituye a un objeto por el otro (Salmina, 2010; Solovieva y Quintanar, 2014). Desde nuestro punto de vista, los ejemplos señalados por los autores se refieren precisamente a las acciones objetales con un fin cultural y no a la función simbólica.

Desde el punto de vista ontogenético, antes de las acciones simbólicas, necesariamente surgen acciones objetales, lo cual permite que surjan sobre su base, las acciones simbólicas. El lenguaje se introduce como un acompañamiento de la realización de las acciones objetales y, más adelante, se abren las posibilidades para la función simbólica y cadenas de juego simbólico complejo. Sin la etapa intermedia de las acciones simbólicas, pareciera ser que la vía del desarrollo del niño fuese: lenguaje y luego función simbólica. Desde nuestro punto de vista, la vía es distinta y más compleja: acciones objetales, lenguaje y luego función simbólica. 
Lo anterior sugiere una gran importancia de introducción de acciones con objetos desde la infancia temprana y la relación genérica de este proceso con la adquisición del lenguaje. Cabe señalar que, actualmente, este punto de vista no es muy común y prevalece una discusión sobre la relación entre lenguaje y la función simbólica. Aún menor atención se ha prestado a la necesidad de evaluación de adquisición de las acciones objetales en la primera fase preescolar, antes de que el niño ingrese a una la institución preescolar formal.

Tanto es así que la mayoría de las pruebas que valoran el desarrollo se centra en la evaluación del desarrollo psicomotor o verbal, así como las habilidades intelectuales a través de las pruebas psicométricas. Existen diversos ejemplos de este tipo de instrumentos como la Escala de Desarrollo Psicomotor de Brunet con adaptaciones de Berum, Barnetche y Alvarado (1967), cuyo objetivo es establecer el cociente de desarrollo; las Escalas Bayley del desarrollo infantil (Bayley, 2006; Albers \& Grieve, 2007); Escala de Uzgiris y Hunt (1975); Inventario de Desarrollo Battelle (Newborg, Stock y Wnek, 1996); Escalas McCarthy (1972) de aptitudes y psicomotricidad para niños; Escala Reynell (1985) para evaluar el desarrollo del lenguaje; Batería Kaufman y Kaufman (1983) para niños, entre otras. Ninguno de estos instrumentos permite caracterizar la dinámica de adquisición de las acciones objetales.

El objetivo de nuestro estudio consiste en mostrar la utilidad de uso de un instrumento de evaluación e interacción cualitativa que permita valorar y caracterizar el grado de adquisición de las acciones con objetos y los procedimientos de juego objetal. Se muestran los resultados que se obtuvieron al aplicarlo en una muestra de niños de entre 13 y 52 meses, observándose la complejización de la ac- tividad objetal hasta llegar al juego simbólico en su etapa inicial.

\section{Participantes}

\section{Materiales y método}

Los participantes del estudio fueron niños mexicanos que asistían a una guardería urbana pública (de la ciudad de Puebla) para trabajadores del estado (nivel sociocultural medio alto). Se seleccionaron a 25 niñas y 27 niños preescolares normales entre 13 y 52 meses de edad, divididos en 3 grupos (muestra de tipo no probabilística o dirigida), como se muestra en la tabla 1.

Los criterios de exclusión del estudio fueron los siguientes:

1. Que presentaran algún trastorno o retraso en el desarrollo diagnosticado y confirmado por médico, psicólogo o psiquiatra.

2. Que presentaran antecedentes de alteraciones o secuelas neurológicas.

3. Que presentaran alguna discapacidad, visual, auditiva o motora.

El tipo de investigación fue exploratorio y descriptivo con un diseño transversal.

\section{Procedimiento}

Se siguieron los procedimientos para garantizar el cumplimiento de los principios y normas éticas de la Declaración de Helsinki para la investigación en seres humanos. De entre estos procedimientos se solicitó el permiso de los padres de los niños por escrito, así como de las educadoras. La muestra se seleccionó a través de una revisión de los expedientes en la guardería. La evaluación del desarrollo objetal se llevó a cabo una sesión individual con cada participante con una duración de $20 \mathrm{mi}$ nutos. 
Para el análisis de resultados se empleó el programa EXCEL y SPSS 15 para Windows. Para cada participante se tomó en cuenta la realización de las acciones objetales.

El análisis de resultados de la ejecución de las acciones objetales se realizó por medio de los porcentajes de ejecuciones de las 6 tareas del protocolo de evaluación elaborado con este propósito.

Se llevó a cabo un análisis de estadística descriptiva, de la cual se obtuvieron: media, desviación estándar y comparaciones entre los grupos por edades. objetales e incluye 6 tareas que se presentan en la tabla 2. En esta se muestran las tareas, las instrucciones y la puntuación que se asigna. Para análisis de los resultados, las puntuaciones se transformaron en porcentajes 0-100.

\section{Resultados}

Se identificó que el $90 \%$ de los participantes tiene una preferencia manual definida. 44 de ellos mostraron preferencia manual derecha y 3 preferencia manual izquierda, mientras que 5 de los participantes mostraron una lateralidad indefinida (usaban ambas manos).

Tabla 1.

Participantes

\begin{tabular}{ccc}
\hline Grupos & Edad & $\begin{array}{c}\text { Número } \\
\text { de niños }\end{array}$ \\
\hline Grupo 1 & 13 a 23 meses & 15 \\
Grupo 2 & 26 a 35 meses & 18 \\
Grupo 3 & 37 a 52 meses & 19 \\
& Promedio de edad & 52 niños \\
\hline
\end{tabular}

\section{Instrumentos}

Para la evaluación de adquisición de las acciones objetales se utilizó el "Protocolo para evaluación de acciones objetales en preescolares menores" (Solovieva y Quintanar, 2014). Se trata de un instrumento cualitativo e interactivo que puede ser fácilmente utilizado para la evaluación del desarrollo, considerando la zona del desarrollo actual y la zona del desarrollo próximo, concepto propuesto por L.S. Vigotsky (Vigotsky, 1984; Solovieva, 2014). El objetivo de este instrumento es valorar el nivel de adquisición de las acciones
Se analizaron los promedios de las puntuaciones obtenidos durante la realización de las tareas del protocolo. De acuerdo a los criterios de cada tarea, el puntaje mayor se multiplica por el número de participantes, obteniendo el $100 \%$. Posteriormente se obtiene la suma de puntuaciones que obtuvieron todos los participantes en cada tarea y se obtiene la proporcionalidad correspondiente (en porcentaje). En este caso, $0 \%$ indica que ninguna de los participantes accede a la tarea, mientras que $100 \%$ indica que todos los menores la logran de manera autónoma. 
Tabla 2.

Tareas de evaluación: tipos de acciones objetales

Tarea

Instrucción

Puntuación

"Muéstrame a qué podemos

1) Acciones objetales espontáneas.

2) Acciones con juguetes con contenido objetal.

3) Acciones con juguetes animados.

4) Sustitución de un objeto por otro.

5) Representación de la acción sin el objeto.

jugar con estos juguetes" (pelotas, muñeca, peine, taza con plato, oso, coche)

"Muéstrame que podemos hacer con estos objetos" mos jugar a los muñecos"

"Vamos a jugar con la muñeca y con el peine, pero ahora la muñeca va a ser el peine y el peine es la muñeca"

"Enséñame como usas la taza, el peine y la toalla"
$0=$ No accede

$1=$ Accede ante realización conjunta

$2=$ Accede con muestra de la acción

$3=$ Realiza independientemente

$0=$ No accede

$1=$ Accede solamente en actividad conjunta

"Muéstrame cómo pode- $2=$ Accede con muestra

$3=$ Accede a la tarea

$4=$ Accede y realiza varias acciones concatenadas con los objetos.

$0=$ No accede

junta

$2=$ Accede con muestra

$3=$ Accede a la tarea

$0=$ No accede

$1=$ Accede solamente en actividad conjunta

$2=$ Accede con muestra

$3=$ Accede a la tarea 
Tabla 2.

Tareas de evaluación: tipos de acciones objetales (continuación).

\begin{tabular}{ll}
\hline & $0=$ No accede \\
6) Representación de acciones & "Enséñame cómo te duer- \\
simbólicas & vidad conjunta \\
& $2=$ Accede con muestra \\
3 & $=$ Accede a la tarea \\
\hline
\end{tabular}

Observaciones generales por cada tarea: presencia o ausencia de las acciones culturales con objetos (objeto real, sustituto o representándolo de manera gestual), variedad de acciones, producción verbal, preferencia manual y ayudas dadas.

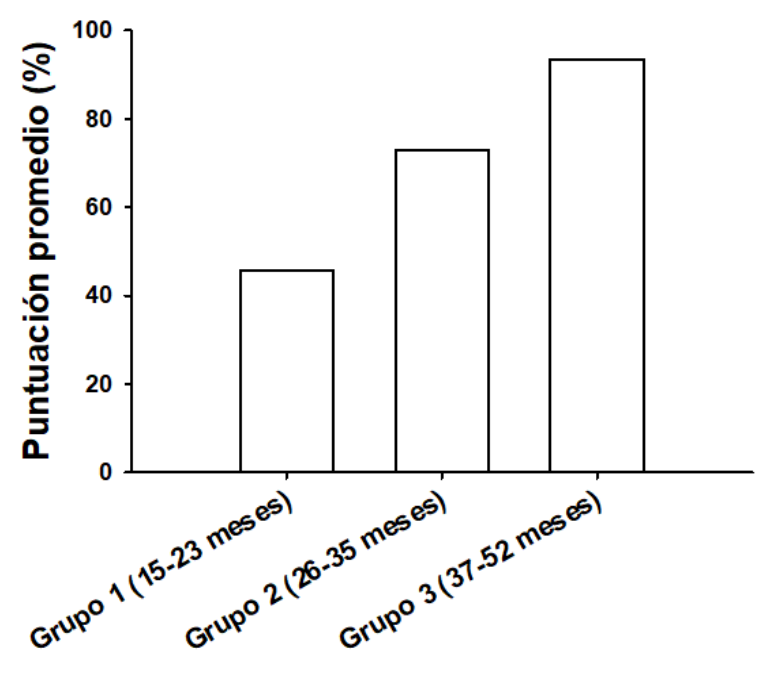

Figura 1. Respuestas promedio (\%) en todas las tareas por grupos de edades.

En la Figura 1 se observa el porcentaje de todas las tareas de acuerdo a la edad de los participantes. Los resultados indican que a mayor edad, los niños acceden a un nivel más complejo de actividad objetal y requieren menor ayuda por parte del adulto.

\section{Análisis por grupos}

A continuación, se presenta en promedio, el porcentaje de niños que respondieron a determinado tipo de respuesta (en orden descendente): 
Grupo 1. En el grupo de los niños menores, entre 15 y 23 meses de edad, se observó que el $48.88 \%$ realizó las tareas con muestra, el $26.71 \%$ no accedió, el $13.38 \%$ lo hizo en colaboración conjunta con el adulto. El $11.16 \%$ accedió sin ayuda. Ningún niño realizó acciones concatenadas (ver Figura 2).

Grupo 2. En el grupo de niños de entre 26 a 35 meses de edad, el $56 \%$ accedió sin ayuda (incluyó el $21 \%$ de los niños que realizaron acciones concatenadas), $25.5 \%$ lo hizo con muestra, el $10.5 \%$ no accedió y el 8.0 $\%$ logró realizar la acción conjunta (ver Figura 3).

Grupo 3. En el grupo de los niños mayores entre 37 y 52 meses de edad, se observó que el 90.4.0\% accedió sin ayuda $\mathrm{y}$ dentro de estos el $44.4 \%$ lo hizo con acciones concatenadas, el $7.6 \%$ lo hizo con muestra, el 1.8 $\%$ no accedió y no hubo ningún niño que lo hiciera en forma de la acción conjunta (ver Figura 4).

\section{Análisis entre grupos}

La comparación de las ejecuciones entre los grupos (ver Figura 5) permite observar que las tareas 1 y 2 fueron realizadas con un promedio por encima del $60 \%$ por todos los grupos. En la tarea 3 de acciones animadas, se observan promedios altos tanto en los grupos segundo (71\%) y

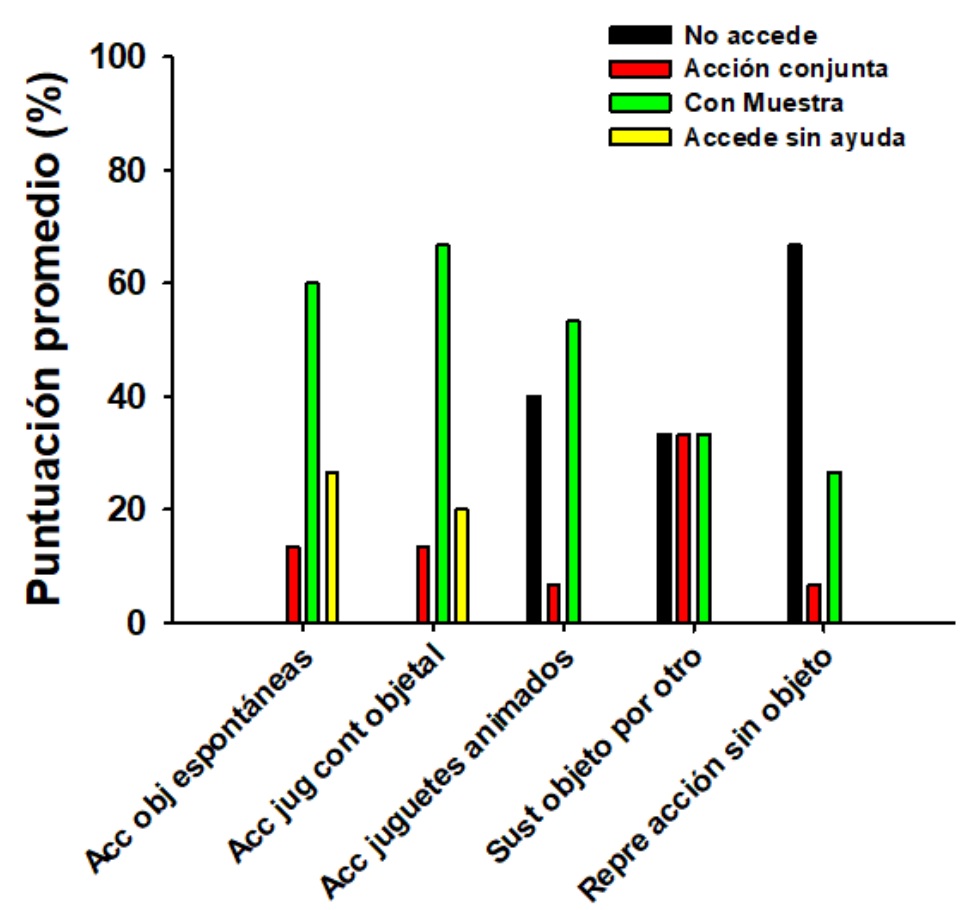

Figura 2. Ejecuciones del grupo 1

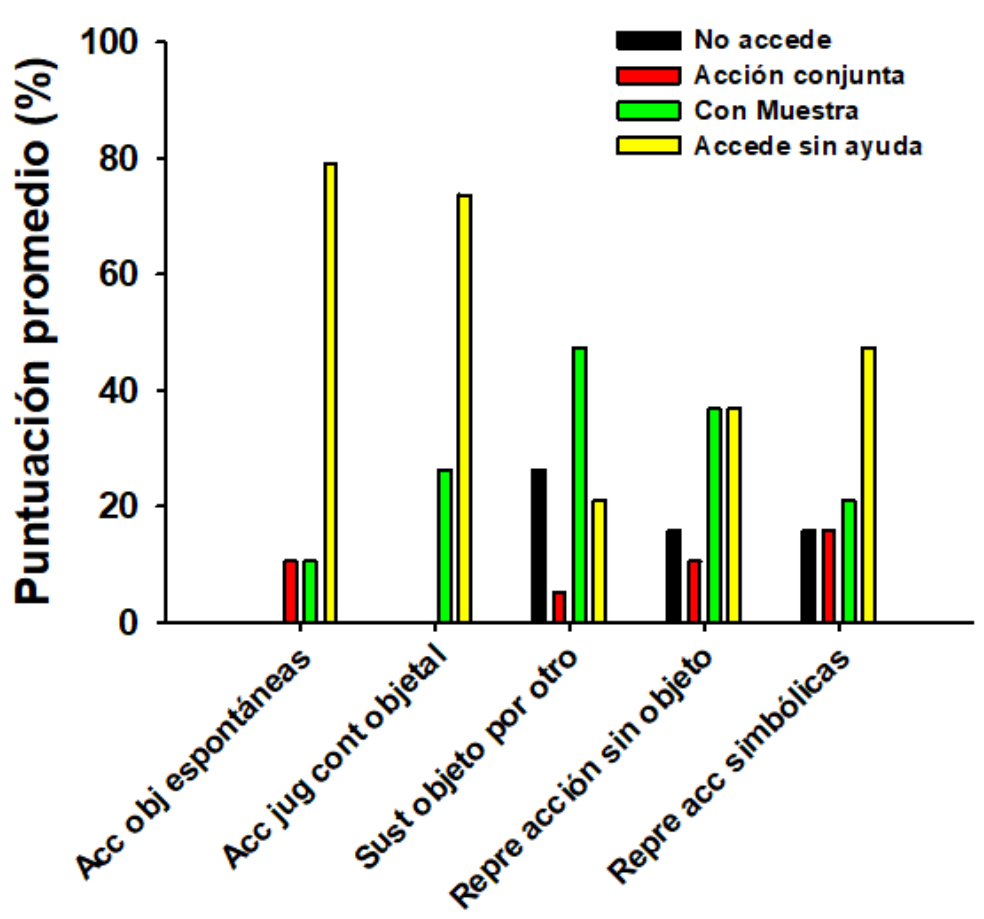

Figura 3. Ejecuciones del grupo 2 
tercero $(83 \%)$. En el grupo de niños menores el promedio fue de $43 \%$.

En la tarea 4, se observa mayor similitud entre los grupos primero y segundo con un promedio de $37 \%$ y $54 \%$ respectivamente, mientras que en el tercer grupo hay un promedio más alto de $87 \%$. Para la tarea 5 , hay diferencias entre los promedios de los grupos. El primer grupo obtuvo un $37 \%$, el segundo grupo un $64 \%$, mientras que el tercero obtuvo un promedio de $96,3 \%$.

En la tarea 6, se observó mayor diferencia entre los promedios de los grupos. El promedio más alto se obtuvo en el tercer grupo (95\%), mientras que en el segundo grupo se obtuvo un promedio de 66,7\%. El nivel más bajo de ejecución independiente se observó en el primer grupo $(20 \%)$.

\section{Discusión}

El objetivo planteado en esta investigación fue caracterizar la actividad objetal en la infancia temprana, en el periodo entre 1 y 3 años de vida. De acuerdo a los resultados obtenidos se identificó que la actividad objetal se va complejizando paulatinamente de acuerdo a la edad (ver Figuras 1 y 5).

Los resultados han mostrado que los niños más pequeños (de 13 a 23 meses) acceden mejor a las acciones con objetos concretos, ya sea con contenido objetal o con juguetes animados (aunque con estos últimos en menor grado). Se trata de uso preferente de objetos inanimados que sugieren manipulación funcional, tales como, objetos de cocina, herramientas, medios de transporte. En esta edad, aun no se observa el juego más complejo, cuando se tienen que llevar a cabo acciones simbólicas con los objetos sustitutos, sin objetos o representando acciones simbólicas (de dormir a un muñeco, por ejemplo). Ningún niño del primer grupo de la población estudiada lo logró hacer de manera independientemente (ver Figura 4).

Entre los niños del segundo grupo de edad, de 26 a 35 meses, además de acceder a las tareas con objetos (con contenido objetal y juguetes animados) de manera más independiente, ya hubo aquellos que accedieron sin ayuda a las acciones simbólicas simples (ver Figura 5). Los niños utilizan los objetos como herramientas funcionales tal y como lo hacen 


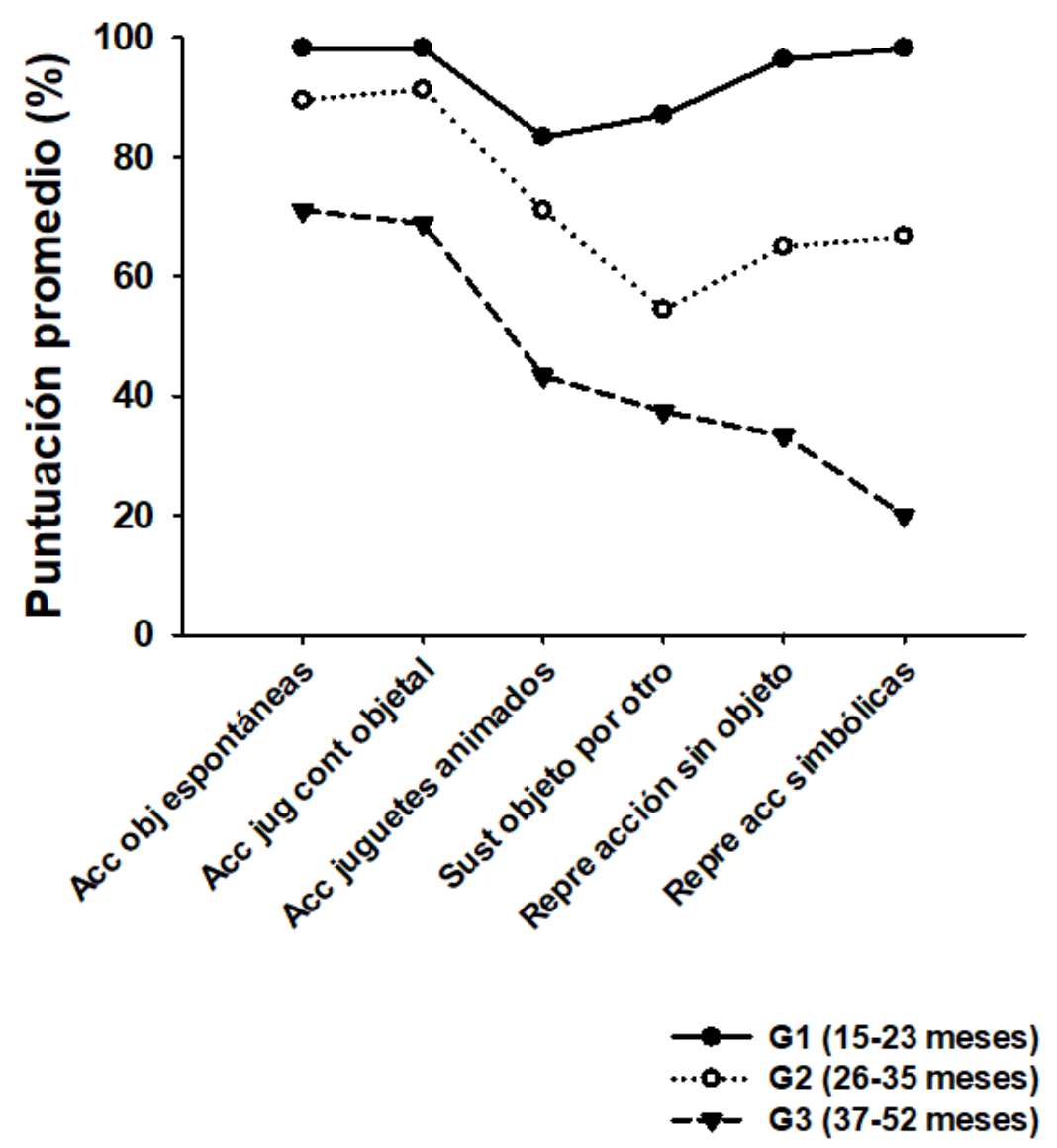

Figura 5. Muestra la comparación de los promedios por tarea entre grupos.

los niños del primer grupo, pero además ya se interesan en relacionar dichas acciones con los "sujetos de estas acciones", es decir, con los juguetes animados (la muñeca come con la cuchara).

Por otra parte, se puede apreciar que prácticamente todos los niños mayores de 37 a 52 meses de edad realizaron todas las tareas propuestas sin necesidad de ayuda, no solo con los objetos, sino también en las acciones simbólicas (sustitución objetal, representación de acciones y acciones representativas) (ver Figura 4). Estos niños logran realizar todas las acciones mencionadas en los grupos anteriores y lo hacen sin necesidad de ayuda por parte del adulto, es decir, no requieren de una muestra del adulto y logran desplegar las acciones objetales ante una animación emocional general del adulto. Además de lo anterior, los niños del tercer grupo de edad logran realizar las sustituciones de un objeto por otro ante animación y la muestra del adulto.

En síntesis, podemos decir que la complejización de la actividad objetal en niños estudiados y su paso gradual a la acción simbólica se da a través de ciertas etapas cualitativas que se describen a continuación.

1) Primera etapa. Se observa un juego con objetos reales con contenido objetal (peine, taza) realizado de forma independien- 
te por el niño. Las acciones se llevan a cabo de manera aislada y muchas veces de manera repetitiva. No hay secuencia de acciones, ni acciones simbólicas.

2) Segunda etapa. Se pasa a un juego con juguetes animados, como el oso y la muñeca, permitiendo realización de una acción objetal de manera repetitiva: por ejemplo, el menor les "da de comer" a los diferentes juguetes animados.

3) Tercera etapa. Los niños juegan con un juguete animado, realizan diferentes acciones con contenido más desplegado y son capaces ya de utilizar objetos sustitutos para los objetos (no animados).

4) Cuarta etapa. Se llega a un juego desplegado, en el cual existe interacción con diversos juguetes animados en forma secuencial (secuencias cotidianas), uso de diversos objetos y de diversos sustitutos de los objetos no animados.

Cabe mencionar que en algunos trabajo se señala la existencia de la relación entre el desarrollo verbal y la acción simbólica, pero no se establecen las etapas de la formación de esta acción y no se menciona la transición de la acción simbólica desde la acción objetal (Lewis, Boucher, Lupton \& Watson, 2000; Palacios, 2009). Para nuestro estudio es importante la posibilidad de establecer la génesis dinámica del desarrollo psicológico del niño en relación con la interacción externa, lo cual nos acerca al concepto de la zona del desarrollo próximo de Vigotsky y sus seguidores (Vigotsky, 1984; Solovieva, 2014). Podemos notar que, mientras que los niños se encuentran en una de las etapas señaladas, la etapa siguiente, normalmente se encuentra en la zona del desarrollo próximo, es decir, los niños realizan las acciones de la etapa siguiente con ayuda e interacción animadora del adulto.
Otro hecho significativo que queremos señalar es el rol que juegan los juguetes animados en la adquisición de formas complejas de juego simbólico. Parece ser que justo la presencia y uso de juguetes animados favorece a la adquisición inicial de la función simbólica en la edad preescolar. Todas estas etapas anteceden al juego temático de roles, que caracteriza a la actividad rectora en la etapa preescolar básica, es decir en la edad de 3 a 6 años (Elkonin, 1987; Solovieva y Quintanar, 2016a). Sin la adquisición de las acciones objetales, el uso de juguetes animados y el inicio de sustitución, es difícil que los niños accedan a la actividad compleja de juego temático de roles sociales, el cual requiere de amplio uso de medios materializados perceptivos y verbales (Solovieva y GonzálesMoreno, 2016).

Estos hallazgos concuerdan con lo que diversos autores señalan acerca de la sustitución de objetos (Galperin, 2009c), con la teoría de las acciones mentales por etapas (Galperin, 2009a; Solovieva, 2014), con las etapas de desarrollo del juego (Elkonin, 1980) y con los tipos de juego de Piaget (1992).

Frádkina (en Elkonin, 1980), al hacer referencia al proceso de sustitución de objetos, menciona que existen dos tipos de trasferencias de acciones. Uno de ellos se refiere a que la acción con un objeto en ciertas condiciones se trasfiere a otras condiciones (un niño que aprende a utilizar el peine en su propia cabeza después peina al caballito, al osito, etc.), pero esto no sucede antes de haber repetido con un objeto la misma acción. Situación semejante fue identificada en los participantes de nuestro estudio, puesto que los niños repetían la acción con un objeto en múltiples ocasiones. Solo después de ello, los menores lograban realizar la misma acción con diversos juguetes animados. La segunda transferencia 
se observa, cuando hay paso al uso de un objeto como sustituto. Frádkina explica que en la primera transferencia hay una generalización de las acciones, mientras que en la segunda ya hay una separación entre el esquema de acción objetal y el uso de objeto concreto.

Elkonin (1980), al referirse a los orígenes del juego en la ontogenia, enuncia que es necesario el desarrollo de los sistemas sensoriales y las coordinaciones sensomotoras para la manipulación y actuación con los objetos. Primero se observan movimientos reiterativos y concatenados, motivados por la novedad de los objetos, lo cual posibilita una actividad exploradora y orientadora. Todos los avances mencionados van de la mano con la orientación constante del adulto, puesto que los niños, en la primera infancia, al participar en el uso social de los objetos junto con los adultos, aprenden su utilización y significación social y, poco a poco, pasan al uso independiente de los mismos.

En nuestra investigación, pudimos identificar que, efectivamente, el niño primero tiene el aliciente por los objetos reales (ver fig. 2). Más adelante los menores son capaces de actuar con objetos sustitutos (ver fig. 3). Finalmente, los niños inician a representar acciones sin objetos, es decir, realizar acciones simbólicas representativas (ver fig. 4), lo cual prepara a la siguiente edad psicológica que es el juego temático de roles (Elkonin, 1980; González y Solovieva, 2015; Solovieva y Quintanar, 2016a).

Es interesante recordar que Piaget $(1984,1992)$ también habla de manera general de estas etapas. En los tipos de juego, este autor indica que inicialmente se da un tipo de juego-ejercicio, en el cual no hay simbolismo; se trata de un juego netamente sensoriomotor y sólo más tarde aparece el juego simbólico. Las diferencias principales entre la postura de
Piaget y los psicólogos de la postura histórico -cultural consisten en el rol del adulto para la orientación del niño en el uso de objetos y en la precisión de las etapas intermedias. Nuestro estudio aporta la importancia de uso obligatorio de juguetes animados introducidos por el adulto que definitivamente favorece a la generalización de las acciones objetales y, a su vez, a la conformación de la función simbólica en la etapa preescolar temprana.

Galperin (2009b) al hablar sobre la formación de las acciones mentales por etapas, refiere que las acciones tienen que llevarse a cabo inicialmente en el plano material o materializado, pasando por la etapa perceptiva, verbal y finalmente llegar al plano mental (Solovieva, Bonilla, y Quintanar, 2008). A partir de esta teoría, podemos plantear que el plano material el niño aprende a utilizar los objetos culturales en referencia a los juguetes animados. Al interaccionar el niño con los objetos reales, las acciones se generalizan y se abstraen (se separan) del objeto. Por ende, el menor logra representar un objeto sin tenerlo ante sí y, de esta forma, pasa a una acción materializada o simbólica inicial que tiene un contenido lúdico. En este plano de acción, el pequeño logra operar no solo con los objetos concretos reales o juguetes, sino también con objetos sustitutos simbólicos de los objetos reales. Es precisamente aquí, cuando ya es palpable la función simbólica, y es cuando el niño ya puede empezar a realizar diferentes tipos de tareas que impliquen el uso de signos y símbolos (dibujo, de actividad constructiva, plástica, de símbolos gráficos, etc.) propios de la edad preescolar (Solovieva y Quintanar, 2013, 2016b; Solovieva, González \& Quintanar, 2016). La sustitución plena representativa de objetos animados se va a realizar más adelante, dentro del contenido de juegos temáticos de roles sociales. 
Por otra parte, Galperin (2009a) refiere que, gracias al uso de los objetos, el niño logra generalizar las características perceptivas (visuales, auditivas, cinestésicas principalmente) para formar imágenes internas. En el momento inicial de este proceso predomina en el infante la relación objeto-acción sobre el significado generalizado. Por ejemplo, el niño considera como taza, aquella que él mismo tiene en casa. Cuando al niño se le van mostrando diversos tipos de tazas con las acciones y verbalizaciones correspondientes por parte adultos, el menor generaliza las características y las acciones con este objeto, condensándose estas en el nombre (significado). Posteriormente la relación se invierte, es decir, comienza a predominar el significado sobre el objeto-acción. Cuando lo anterior sucede, se da la sustitución de los objetos y la representación de acciones, dando origen así a la acción simbólica (Vigotsky, 1979).

Para Salmina (2010), el desarrollo simbólico complejo es una condición necesaria e indispensable para la posterior adquisición de la lecto-escritura y el cálculo. Podemos hablar de una forma inicial de la acción simbólica o acción simbólica lúdica, lo cual se debe diferenciar de acciones materializadas intelectuales o lógicas complejas que se pueden adquirir en las etapas posteriores del desarrollo preescolar y escolar (Talizina, 2009; Salmina, 2010). La acción objetal, en este sentido, aparece como una plataforma genética en el desarrollo de la acción simbólica.

\section{Conclusiones}

Los datos que arroja la investigación se relacionan con la posibilidad de identificación de las etapas cualitativas en la adquisición de las acciones con los objetos concretos en niños en la edad preescolar temprana, es decir, en la edad entre 1 y 3 años de edad. Estos datos pueden ser de importancia para especialistas e instituciones que trabajen con población infantil normal o con alteraciones en el neurodesarrollo, debido a que permite observar etapas de adquisición de acciones objetales. Es importante señalar que en las etapas iniciales las acciones objetales se encuentran en la zona del desarrollo próximo, es decir, se realizan con ayuda del adulto. En las siguientes etapas, mismas acciones se encuentran en la zona del desarrollo actual. Estos datos pueden indicar las estrategias interventivas en aquellos niños que no logran realizar las acciones objetales después de la edad de 1 año. El protocolo utilizado puede ser de utilidad para evaluación del desarrollo en la edad preescolar temprana para caracterizar cualitativamente el nivel del desarrollo psicológico del niño y señalar las recomendaciones para cada caso particular. Las investigaciones futuras deben mostrar las posibilidades de intervención en casos de niños con diversos trastornos en esta edad, así como establecer relación con el desarrollo verbal, estrategias de interacción familiar y las etapas futuras del desarrollo simbólico.

\section{Referencias}

Albers, C. A. \& Grieve, A. J. (2007). Test review: Bayley, N. Bayley scales of infant and toddler development-third edition. San Antonio, TX: Harcourt assessment. Journal of Psychoeducational Assessment. 25 (2): 180-90. doi: $10.1177 / 0734282906297199$

Bates, E., Benigni, L., Bretherton, I., Camaioni, 1., \& Volterra, V. (1979). The emergence of symbols: Cognition and Communication. In infancy. New York: Academic Press.

Bayley, N. (2006). Bayley scales of infant and toddler development (3rd ed.). San Antonio, TX: Pearson.

Berum, T., Barnetche, L. y Alvarado, M. (1967). Escala para medir el desarrollo psicomotor del niño mexicano. Batería BBA. México, D.F: IMSS 
Bonilla, M. R., Solovieva, Y. y Jiménez, N. (2012). Valoración del nivel del desarrollo simbólico en la edad preescolar. Revista CES Psicología, 5 (2), 56-69. http://revistas.ces.edu.co/index.php/ psicologia/article/view/2249

Cruz, L. (1995). Selección de lecturas de psicología infantil. La Habana: Editorial Pueblo y Educación.

Davidov, V.V. (1988). Problemas del desarrollo psíquico de los niños. La enseñanza escolar y el desarrollo psíquico. Moscú: Editorial Progreso.

Elkonin, D. B. (1980). Psicología del juego. España: Visor.

Elkonin, D. B. (1987) Sobre el problema de la periodización del desarrollo psíquico en la infancia. En D. Davidov \& M. Shuare, (Eds.), La psicología evolutiva y pedagógica en la URSS (pp. 104123). Moscú: Progreso.

Galperin, P. Ya. (2009a). La formación de las imágenes sensoriales y de los conceptos. En L. Quintanar y Y. Solovieva (Comp.), Las funciones psicológicas en el desarrollo del niño (pp. 6475). México: Trillas.

Galperin, P. Ya. (2009b). La investigación del desarrollo intelectual del niño. En L. Quintanar y Y. Solovieva (Comp). Las funciones psicológicas en el desarrollo del niño. (pp. 98-112). México: Trillas.

Galperin, P. Ya. (2009c). Acerca del lenguaje interno. En L. Quintanar y Y. Solovieva. (Comp.), Las funciones psicológicas en el desarrollo del niño. (pp. 91-97). México: Trillas.

González, C. X. y Solovieva, Y. (2014). Propuesta de método para el estudio de la formación de la función simbólica en la edad infantil. Tesis Psicológica, 9 (2), 58-79 http://www.redalyc.org/ articulo.oa? id=139039784005

González, C.X. y Solovieva, Y. (2015). Indicadores de adquisición de la fun-ción simbólica en el nivel de acciones materializadas en preescolares. Pensamiento Psicológico, 13 (2), 79-94. doi:10.11144/Javerianacali.PPSI13-2.iafs

González, C. X. y Solovieva, Y. (2016). Caracterización del nivel de desarrollo de la función simbólica en niños preescolares. Rev. CES Psicol., 9 (2), 80-99. doi: 10.21615/cesp.9.2.6

Kaufman, A. S., \& Kaufman, N. L. (1983). Kaufman Assessment Battery for Children [Database record]. Retrieved from PsycTESTS. doi:10.1037/ t27677-000

Lewis, V., Boucher, J., Lupton, L., \& Watson, S. (2000). Relationships between symbolic play, functional play, verbal and nonverbal ability in young children. International Journal of Language and Communication Disorders, 35, 117 127. doi:10.1080/136828200247287

Lisina, M. (1986). La comunicación con los adultos en los niños hasta los siete años de vida. En: I. I. Iliasov y V. Ya Liaudis (Eds.), Antología de la psicología pedagógica y de las edades (pp. 125131). La Habana: Pueblo y Educación.

Liublinskaia, A. A. (1971). Desarrollo psíquico del niño. México: Grijalbo.

McCarthy, D. (1972). Manual for the McCarthy Scales of Children's Abilities. New York: Psychological Corp.

McCune-Nicolich, L. (1995). A normative study of representational play at the transition to language. Developmental Psychology, 31(2), 198-206.

Moro, C., y Rodríguez, C. (1991). ¿Por qué el niño tiende el objeto hacia el adulto? La construcción social de los objetos. Infancia y Aprendizaje, 14 (53), 99-118. doi:10.1080/02103702.1991.10822286

Newborg, J., Stock, J. R., y Wnek, L. (1996). Inventario de Desarrollo Battelle. Madrid: TEA.

Palacios, P. (2009). Origen de los usos simbólicos de los objetos en los niños en contextos de comunicación e interacción triádicos. Tesis doctoral inédita. Universidad Autónoma de Madrid. Madrid, España.

Piaget, J. (1992). Seis estudios de psicología. México: Ariel.

Piaget, J. (1984). Psicología del niño. Madrid: Morata.

Piaget, J. y Inhelder, B. (1997). Psicología del niño. México: Ediciones Morata.

Reynell, J. K. (1985). Escala Reynell. para evaluar el desarrollo del lenguaje. Madrid: MEPSA.

Salmina, N. (2010). La función semiótica y el desarrollo intelectual. En Y. Solovieva \& L. Quintanar (Eds.), Antología del desarrollo psicológico del niño en edad preescolar. (pp. 75-84). México: Trillas.

Slade, A. (1987). A Longitudinal Study of Maternal Involvement and Symbolic Play during the Toddler Period. Child Development, 58(2), 367375. doi: 10.2307/1130513

Solovieva, Y., Bonilla, M., y Quintanar, L. (2008). Aproximación histórico-cultural: Intervención en los trastornos del aprendizaje. En J. Eslava (et al.) Los trastornos del aprendizaje: Perspectivas neuropsicológicas (pp. 227-266). Bogotá: Cooperativa Editorial Magisterio, Instituto Colombiano de neurociencias, Benemérita Universidad Autónoma de Puebla. 
Solovieva, Y. y Quintanar, L. (2016a). La actividad de juego en la edad preescolar. México: Trillas.

Solovieva, Y. y Quintanar, L. (2016b). Dibujo como la actividad formativa. México: Trillas.

Solovieva, Y. y Quintanar, L. (2013). Evaluación del desarrollo simbólico en niños preescolares mexicanos. Cultura y Educación, 25(2), 167-182. doi: 10.1174/113564013806631273

Solovieva, Y. y Quintanar, L. (2014). Evaluación del desarrollo para niños preescolares menores. México: Universidad Autónoma de Puebla.

Solovieva, Y. y Gonzáles-Moreno, C. (2016). Indicadores de la adquisición de la función simbólica en el nivel de acciones verbales en preescolares. Revista Fac. Med. 64 (2): 257-265. doi:10.15446/revfacmed.v64n2.52995

Solovieva, Y., Gonzáles, C. \& Quintanar, L. (2016). Developmental analysis of symbolic perceptual actions in preschools. British Journal of Education, Society \& Behavioural Science. 15 (3): 113. doi : 10.9734/BJESBS/2016/24459

Solovieva, Y. (2014). Desarrollo de la actividad desde el paradigma histórico-cultural. México: CEIDE.

Talizina, N.F. (2009). La teoría de la actividad aplicada a la enseñanza. México: Universidad Autónoma de Puebla.

Tomasello, M. (2013). Los orígenes de la comunicación humana. Buenos Aires: Katz.

Uzgiris, I. C., \& Hunt, J. McV. (1975). Assessment in infancy. Chicago: University of Illinois Press.

Vigotsky, L. S. (1979). El papel del juego en el desarrollo del niño (Trad. S. Furio.). En M. Cole, V. Jhon-Steiner, S. Scribner \& E. Souberman (Eds.), El desarrollo de los procesos psicológicos superiores (pp.141-158). Barcelona: Critica. (Trabajo original publicado en 1933).

Vigotsky, L.S. (1984). Obras Escogidas. Tomo 4. Moscú: Pedagogía. 